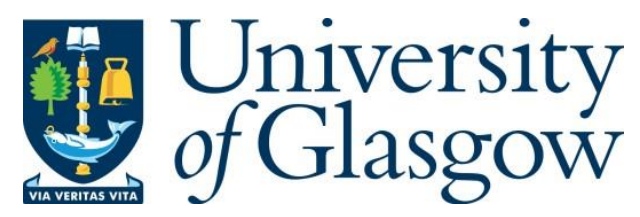

Wolz, T., McLellan, L., Schneider, A., Stehli, A., Brehm, J. D., Rotzinger, H., Ustinov, A. V. and Weides, M. (2021) Frequency fluctuations of ferromagnetic resonances at millikelvin temperatures. Applied Physics Letters, 119(21), 212403.

There may be differences between this version and the published version. You are advised to consult the publisher's version if you wish to cite from it.

http://eprints.gla.ac.uk/259344/

Deposited on: 22 November 2021

Enlighten - Research publications by members of the University of Glasgow http://eprints.gla.ac.uk 


\section{Frequency fluctuations of ferromagnetic resonances at milliKelvin temperatures}

Tim Wolz, ${ }^{1}$ Luke McLellan, ${ }^{2}$ Andre Schneider, ${ }^{1}$ Alexander Stehli, ${ }^{1}$ Jan David Brehm, ${ }^{1}$ Hannes Rotzinger,,${ }^{1,3}$ Alexey V. Ustinov, ${ }^{1,4,5}$ and Martin Weides ${ }^{2}$

${ }^{1)}$ Institute of Physics, Karlsruhe Institute of Technology, 76131 Karlsruhe, Germany

2) James Watt School of Engineering, Electronics \& Nanoscale Engineering Division, University of Glasgow, Glasgow G12 8QQ,

United Kingdom

${ }^{3)}$ Institute for Quantum Materials and Technologies, Karlsruhe Institute of Technology, 76131 Karlsruhe, Germany

${ }^{4)}$ National University of Science and Technology MISIS, 119049 Moscow, Russia

5) Russian Quantum Center, 143025 Skolkovo, Moscow, Russia

(*Electronic mail: martin.weides@glasgow.ac.uk)

(Dated: 24 October 2021)

Unwanted fluctuations over time, in short, noise, are usually detrimental to device performance, especially for quantum coherent circuits. Recent efforts have demonstrated routes to utilizing magnon systems for quantum technologies by interfacing single magnons with superconducting qubits. However, the coupling of several components often introduces additional noise to the system, hence degrading its coherence. Researching the system's temporal behavior can help to identify the underlying noise sources, which is a vital step for improving coherence times and hybrid device performance. Yet, the frequency noise of the ferromagnetic resonance (FMR) has so far been unexplored at mK-temperatures. Here, we investigate such FMR frequency fluctuations of a YIG sphere and find that these fluctuations are independent of temperature and drive power. This suggests that the measured frequency noise in YIG is dominated by undetermined noise sources, whose properties are not consistent with the conventional model of two-level systems, despite their effect on the sample linewidth. Moreover, the functional form of the FMR frequency noise power spectral density (PSD) cannot be described by a simple power law. By employing time-series analysis, we find a closed function for the PSD that fits to the observations. Our results underline the necessity of coherence improvements to magnon systems for useful applications in quantum magnonics.

Fluctuations of the resonance frequency and other forms of noise can drastically hamper the performance of sensors, amplifiers, and information processing circuits. This is accurate at room temperature but particularly crucial for quantum devices, where environmental noise leads to decoherence. The recent coupling of single magnons to superconducting qubits ${ }^{1-3}$ and resonators ${ }^{4-6}$ has lead to research on hybrid quantum magnonics ${ }^{7-9}$. There, the goal is a combination of quantum computing's exponential speedup with magnonics, 10,11 versatile low-loss devices. A radio frequency-to-light conversion based on magnons ${ }^{12-14}$ presents a possible direction towards a quantum internet, but it also requires a coupling of several quantum systems. Such a coupling often results in additional loss channels and increased noise, which presents a major obstacle in quantum magnonics ${ }^{15}$. Yet, the influence and origin of magnonic noise is still largely an open question. So far, the phase noise of magnetic tunnel junction oscillators ${ }^{16,17}$, the amplitude and phase noise of a magnonic waveguide ${ }^{18,19}$ at room temperature, and the magnetization noise of spins ${ }^{20-22}$ from a theoretic viewpoint have been explored. At room temperature, YIG oscillators are known to exhibit extremely low phase noise $\mathrm{e}^{23-25}$. At $\mathrm{mK}$ temperatures however, frequency fluctuations of the ferromagnetic resonance (FMR) have eluded attention, despite the recent reports of an increased linewidth due to two-level systems (TLS) ${ }^{26-28}$.

Here, we experimentally observe such FMR frequency fluctuations with a focus on an yttrium-iron-garnet (YIG). After an introduction to the measurement setup and the spec- troscopic characterization of the YIG sample, we present the results of the fluctuation measurements and analyze them in the frequency- as well as time domain.

Our experimental setup (Fig. 1(a)) consists of a vector network analyzer (VNA) connected to the magnetic medium via a strip-line in a notch-type configuration. The sample, a YIG sphere with diameter $d=0.2 \mathrm{~mm}$, is mounted in a solenoid coil inside a dilution refrigerator. The VNA offers a straightforward procedure for frequency noise measurements. Sweeping the probe frequency allows for a characterization of the sample via its $S_{i j}(\omega)$-matrix element, from which we extract the FMR linewidth. Then, to measure frequency fluctuations, we employ the continuous wave mode of the VNA with probe frequency $\omega_{\mathrm{p}}$. Here, we record a time trace of the sample's frequency response at one single point close to resonance $\left(\omega_{\mathrm{p}} \approx \omega_{\mathrm{r}}\right)$. Fluctuations in the phase $\arg S_{21}$ can then be converted to resonance frequency fluctuations via the slope in the linear region of $\arg S_{21}(\omega)$, see Fig. 1 (b) for a schematic overview and supplementary material I for more details. All measurements are performed and evaluated with the open-source measurement suite qkit ${ }^{30}$.

We start with the spectroscopic characterization of our sample. The FMR is tuned to $\omega_{\mathrm{r}} / 2 \pi=6.11 \mathrm{GHz}$, corresponding to an external field $\mu_{0} H \approx 0.21 \mathrm{~T}$, where the sample is fully magnetized. Figures $1(\mathrm{c}, \mathrm{d})$ show the amplitude and phase of the background-corrected complex $S_{21}$ frequency response. A circle fit ${ }^{29}$ returns the internal linewidth (HWHM) $\kappa_{\mathrm{i}}=\omega_{\mathrm{r}} /\left(2 Q_{i}\right)$, with $Q_{\mathrm{i}}$ as internal Q-factor. Varying power, $P$, and temperature, $T$, we find a linewidth dependence that 


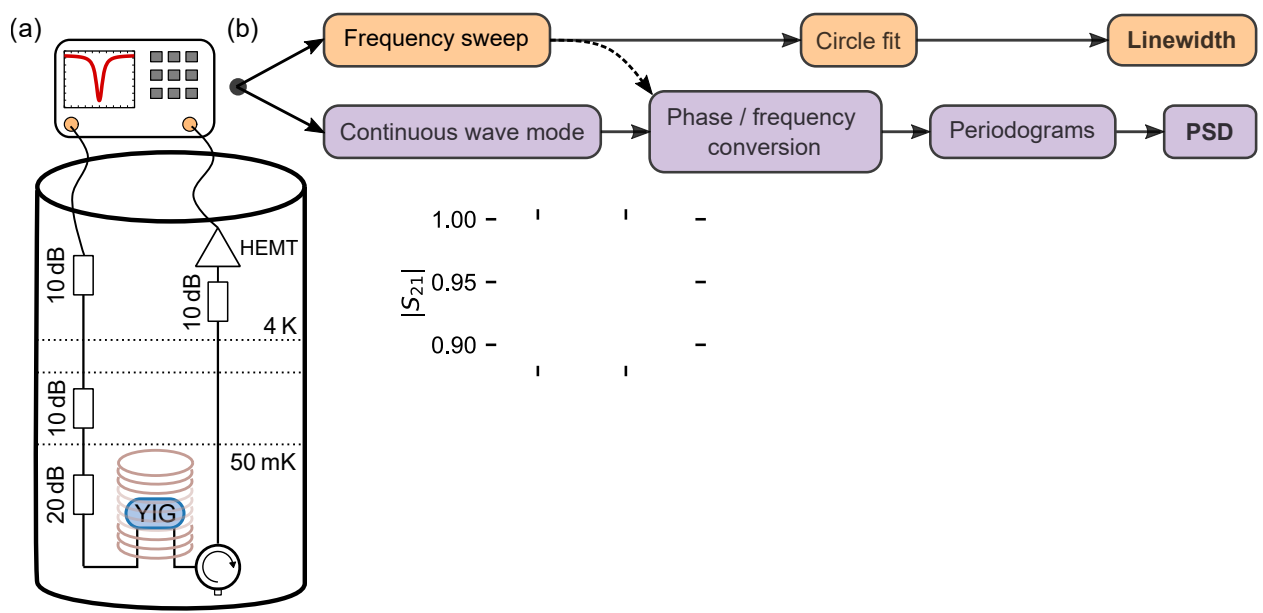



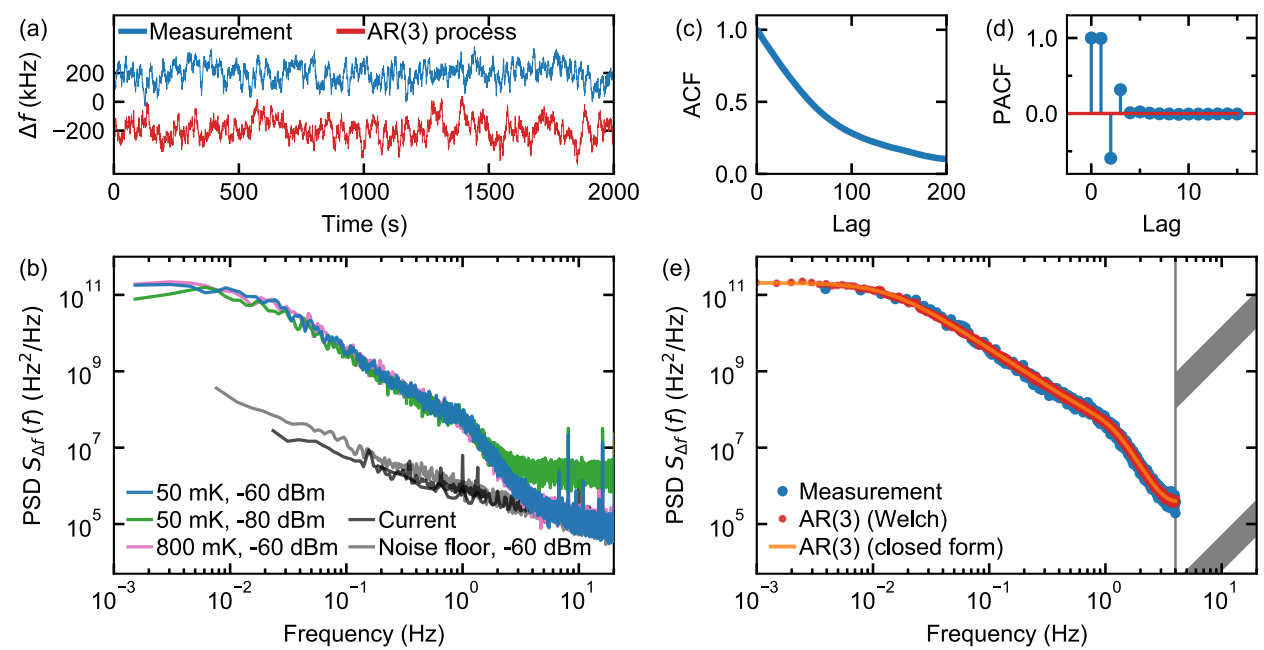

FIG. 2. Frequency fluctuations of a ferromagnetic resonance (FMR) at mK temperatures. (a) A segment of the measured time trace of the frequency fluctuations $(P=-60 \mathrm{dBm}, T=50 \mathrm{mK})$ compared to generated data, a realization of a third order autoregressive process AR(3). For better visibility the data are offset by $\pm 200 \mathrm{kHz}$ and the measured time trace is post averaged to $8 \mathrm{~Hz}$, so that periodic signals are removed. (b) Power spectral density (PSD) of FMR frequency fluctuations for different input powers and temperature (without post averaging). Below $3 \mathrm{~Hz}$, the sample noise PSD exceeds the amplitude of parasitic noise sources, such as the HEMT amplifier and the current source. No dependence on these external parameters can be observed in the low frequency range of the PSD. The functional form of the PSDs is close to a Lorentzian but shows a steeper decay at around $1 \mathrm{~Hz}$. (c,d) Autocorrelation (ACF) and partial autocorrelation (PACF) of the time trace data displayed in (a). PACF only shows values significantly different from zero up to lag $n=3$, indicating an $\operatorname{AR}(3)$ process. Note the different scales of the $\mathrm{x}$-axes. (e) A comparison of measured FMR noise data with an AR(3) process shows excellent agreement. Shaded area is due to the resampling to $8 \mathrm{~Hz}$ for the time series analysis. For the generated data, the PSD is calculated via Welch's method and via Eq. (4) with estimated coefficients.

of the noise measurements, which are presented in Fig. 2 Panel (a) shows a recorded time trace of the FMR frequency fluctuations, which is used to evaluate the PSD, as displayed in Fig. 2 (b). We note that the observed frequency noise PSD is indeed higher than the noise floor of the setup, given by the HEMT amplifier. It is also higher than the noise produced by the current source and higher than measured FMR frequency noise at room temperature (see supplementary material IC). We also observe a functional form of the PSD that does not fit a simple power law. Up to $1 \mathrm{~Hz}$, it can be described by a Lorentzian function but then a steep decrease follows. To test the influence of external parameters, we vary the temperature from $50 \mathrm{mK}$ to $800 \mathrm{mK}$ and sweep the input power around the critical power from $-60 \mathrm{dBm}$ down to $-100 \mathrm{dBm}$. Three curves are shown as examples, see supplementary material II for more data. The frequency noise PSDs all show an independence of temperature and power. The increased white noise part for the low power PSD arises from amplifier noise. Taking all these points together, we conclude that TLS as described by the standard or generalized tunneling model are not the most dominant noise source for frequency fluctuations in our magnetic system. The lack of a power dependence is the strongest argument. A comparison to superconducting resonators ${ }^{37}$ supports this statement. There, the TLS noise PSD at $0.1 \mathrm{~Hz}$ is three magnitudes lower than the observed FMR fluctuation PSD. Hence, despite showing a power or temperature dependent resonance linewidth, undetermined noise sources most likely mask the influence of TLS noise in the magnon system.

As the measured PSD does not follow a simple power law, a quantitative analysis is difficult. As a starting point for future works, we present a method to find a closed function for FMR frequency noise. This method is closely related to maximum entropy spectral analysis ${ }^{41}$, and based on time series analysis. There, a basic model describing random data is the autoregressive (AR) process, defined as

$$
y_{t}=\varepsilon_{t}+\sum_{i=1}^{p} a_{i} y_{t-i}
$$

A random data point $y_{t}$ at time $t$ is calculated via a weighted sum of the last $p$ data points with free parameters $a_{i}$ plus a white noise term with a Gaussian probability density function $\mathscr{N}(\sigma, \mu=0) . \quad \sigma$ and $\mu$ denote the standard deviation and mean value, respectively. See supplementary material III A for further information about AR processes. Importantly, these processes can be described by the following 
closed function ${ }^{42}$

$$
S(f)=\frac{2 \sigma^{2} \delta t}{\mid 1-\sum_{k=1}^{p} a_{k} \mathrm{e}^{-i 2 \pi k \delta t f \mid}} .
$$

Hence, our goal is to estimate the order $p$ and the $a_{i}$. For this purpose, we make use of the autocorrelation function (see Fig. 2 (c)), showing an exponential-like decay and hence pointing towards an AR process, as expected from Eq. (3). The partial autocorrelation function (PACF), displayed in Fig. 2(d), gives only the direct correlation between data points, i.e., the indirect propagation of a perturbation over other data points is switched off. We can therefore estimate the order $p=3$ from the number of values significantly different from zero. The $a_{i}$ coefficients can then be calculated by employing the Yule-Walker equations ${ }^{43,44}$, which relate the ACF to the $a_{i}$ (supplementary material III B). The estimated coefficients are $a_{1}=1.764, a_{2}=-1.079, a_{3}=0.309, \sigma=$ $5.284 \mathrm{kHz}$. Note that the order and subsequently the coefficients depend on the chosen sampling rate. For the time series analysis, a digital post averaging to a sampling frequency of $8 \mathrm{~Hz}$ was performed, removing periodic signals and the $1 / f$ amplifier part. These noise forms are not captured by an AR process. See supplementary material IV for different sampling rates. With the estimated values, we can generate a model time trace for comparison (Fig. 2(a)) and calculate its PSD Figure 2 (e) shows an excellent agreement of the measured PSD with both the numerical simulation and the closed form, confirming the validity of the AR model. Furthermore, from the estimated parameters and the ACF, we can conclude that FMR frequency fluctuations show a Brownian motion like behavior. Yet these fluctuations are exponentially damped out over time and require a weighted sum of data points in the past. The weighted sum indicates several noise mechanisms that occur on different timescales ${ }^{21}$. Regarding the PSD, a damped Brownian motion would be one process that leads to the dominating Lorentzian part below $1 \mathrm{~Hz}$. Since also other mechanisms exist that manifest in a Lorentzian PSD, such as random telegraphic noise or a modulation of the measure data, more work is necessary to completely understand the shape of the PSD and to identify the underlying noise mechanisms. Exploring FMR noise in a variety of magnetic materials, different sample geometries and fabrication procedures would be advantageous.

In conclusion, we studied FMR frequency fluctuations at $\mathrm{mK}$ temperatures. The recorded PSDs do not show a simple power law. They are independent of temperature and input power, which indicates undetermined noise mechanisms stronger than the influence of TLS described by the the generalized / standard tunneling model. We also presented a method to analyze noise data in the time domain. This is especially useful if a simple power law is not sufficient to describe the noise PSD. With this method and after post averaging of the data down to $8 \mathrm{~Hz}$, we find an excellent agreement of the measured data with an $\mathrm{AR}(3)$ process, suggesting that several noise processes on different time scales are at play. A comparison to room temperature measurements has shown increased noise at low temperatures, underpinning the importance of improving magnon coherence for useful applications in the quantum limit. With this work, we hope to spark a broader interest into magnon decoherence research.

\section{SUPPLEMENTARY MATERIAL}

See supplementary material for more information about the experimental details, including measurements of parasitic noise sources, room temperature noise measurements with YIG and lithium ferrite, and additional explanations about the time series analysis.

\section{ACKNOWLEDGMENTS}

We wish to acknowledge fruitful discussions with Jürgen Lisenfeld, Khalil Zakeri, Dmytro Bozhko, and Mehrdad Elyasi. We acknowledge financial support from the former Helmholtz International Research School for Teratronics (TW), the Landesgraduiertenförderung (LGF) BadenWürttemberg (A St), the Carl-Zeiss-Foundation (A Sch) and Studienstiftung des Deutschen Volkes (JDB). This work was supported by the European Research Council (ERC) under the Grant Agreement 648011 (MW) and by the Ministry of Science and Higher Education of the Russian Federation in the framework of the State Program (Project No. 0718-20200025) (AVU).

\section{DATA AVAILABILITY STATEMENT}

The data that support the findings of this study are available from the corresponding author upon reasonable request.

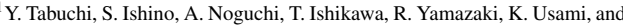
Y. Nakamura, "Coherent coupling between a ferromagnetic magnon and a superconducting qubit," Science 349, 405-408 (2015).

D. Lachance-Quirion, Y. Tabuchi, S. Ishino, A. Noguchi, T. Ishikawa, R. Yamazaki, and Y. Nakamura, "Resolving quanta of collective spin excitations in a millimeter-sized ferromagnet," Science Advances 3, e1603150 (2017)

D. Lachance-Quirion, S. P. Wolski, Y. Tabuchi, S. Kono, K. Usami, and Y. Nakamura, "Entanglement-based single-shot detection of a single magnon with a superconducting qubit," Science 367, 425-428 (2020). ${ }^{4}$ R. G. E. Morris, A. F. van Loo, S. Kosen, and A. D. Karenowska, "Strong coupling of magnons in a YIG sphere to photons in a planar superconducting resonator in the quantum limit," Scientific Reports 7, 11511 (2017).

${ }^{5}$ Y. Li, T. Polakovic, Y.-L. Wang, J. Xu, S. Lendinez, Z. Zhang, J. Ding, T. Khaire, H. Saglam, R. Divan, J. Pearson, W.-K. Kwok, Z. Xiao, V. Novosad, A. Hoffmann, and W. Zhang, "Strong Coupling between Magnons and Microwave Photons in On-Chip FerromagnetSuperconductor Thin-Film Devices," Physical Review Letters 123, 107701 (2019)

P. G. Baity, D. A. Bozhko, R. Macêdo, W. Smith, R. C. Holland, S. Danilin, V. Seferai, J. Barbosa, R. R. Peroor, S. Goldman, U. Nasti, J. Paul, R. H. Hadfield, S. McVitie, and M. Weides, "Strong magnon-photon coupling with chip-integrated YIG in the zero-temperature limit," arXiv:2104.08068 [cond-mat, physics:physics] (2021).

D. Lachance-Quirion, Y. Tabuchi, A. Gloppe, K. Usami, and Y. Nakamura, "Hybrid quantum systems based on magnonics," Applied Physics Express 12, 070101 (2019) 
${ }^{8}$ Y. Li, W. Zhang, V. Tyberkevych, W.-K. Kwok, A. Hoffmann, and V. Novosad, "Hybrid magnonics: Physics, circuits, and applications for coherent information processing," Journal of Applied Physics 128, 130902 (2020).

${ }^{9}$ M. Elyasi, Y. M. Blanter, and G. E. W. Bauer, "Resources of nonlinear cavity magnonics for quantum information," Physical Review B 101, 054402 ity magn

(2020).

${ }^{10}$ V. V. Kruglyak, S. O. Demokritov, and D. Grundler, "Magnonics," Journal of Physics D: Applied Physics 43, 264001 (2010).

${ }^{11}$ A. V. Chumak, V. I. Vasyuchka, A. A. Serga, and B. Hillebrands, "Magnon spintronics," Nature Physics 11, 453-461 (2015)

${ }^{12}$ R. Hisatomi, A. Osada, Y. Tabuchi, T. Ishikawa, A. Noguchi, R. Yamazaki, K. Usami, and Y. Nakamura, "Bidirectional conversion between microwave and light via ferromagnetic magnons," Physical Review B 93 , 174427 (2016).

${ }^{13}$ A. Osada, R. Hisatomi, A. Noguchi, Y. Tabuchi, R. Yamazaki, K. Usami, M. Sadgrove, R. Yalla, M. Nomura, and Y. Nakamura, "Cavity Optomagnonics with Spin-Orbit Coupled Photons," Physical Review Letters 116, 223601 (2016)

${ }^{14}$ X. Zhang, N. Zhu, C.-L. Zou, and H. X. Tang, "Optomagnonic Whispering Gallery Microresonators," Physical Review Letters 117, 123605 (2016).

${ }^{15}$ A. A. Clerk, K. W. Lehnert, P. Bertet, J. R. Petta, and Y. Nakamura, "Hybrid quantum systems with circuit quantum electrodynamics," Nature Physics 16, 257-267 (2020)

${ }^{16}$ D. Houssameddine, U. Ebels, B. Dieny, K. Garello, J.-P. Michel, B. Delaet, B. Viala, M.-C. Cyrille, J. A. Katine, and D. Mauri, "Temporal Coherence of $\mathrm{MgO}$ Based Magnetic Tunnel Junction Spin Torque Oscillators," Physical Review Letters 102, 257202 (2009).

${ }^{17}$ M. Quinsat, D. Gusakova, J. F. Sierra, J. P. Michel, D. Houssameddine, B. Delaet, M.-C. Cyrille, U. Ebels, B. Dieny, L. D. Buda-Prejbeanu, J. A. Katine, D. Mauri, A. Zeltser, M. Prigent, J.-C. Nallatamby, and R. Sommet, "Amplitude and phase noise of magnetic tunnel junction oscillators," Applied Physics Letters 97, 182507 (2010).

${ }^{18}$ S. Rumyantsev, M. Balinskiy, F. Kargar, A. Khitun, and A. A. Balandin, "The discrete noise of magnons," Applied Physics Letters 114, 090601 (2019)

${ }^{19}$ S. Rumyantsev, M. Balinskiy, F. Kargar, A. Khitun, and A. A. Balandin, "Amplitude and Phase Noise of Magnons," arXiv:1909.00085 [cond-mat, physics:physics] (2019)

${ }^{20}$ R. Kubo and N. Hashitsume, "Brownian Motion of Spins," Progress of Theoretical Physics Supplement 46, 210-220 (1970).

${ }^{21} \mathrm{~K}$. Miyazaki and K. Seki, "Brownian motion of spins revisited," The Journal of Chemical Physics 108, 7052-7059 (1998).

${ }^{22}$ J. Foros, A. Brataas, G. E. W. Bauer, and Y. Tserkovnyak, "Noise and dissipation in magnetoelectronic nanostructures," Physical Review B 79, 214407 (2009).

${ }^{23}$ A. Khanna and J. Buenrostro, "2-22 GHz low phase noise silicon bipolar YIG tuned oscillator using composite feedback," in 1992 IEEE MTT-S Microwave Symposium Digest (1992) pp. 1297-1299 vol.3

${ }^{24}$ D. Eliyahu and L. Maleki, "Tunable, ultra-low phase noise YIG based optoelectronic oscillator," in IEEE MTT-S International Microwave Symposium Digest, 2003, Vol. 3 (2003) pp. 2185-2187 vol.3.

${ }^{25}$ A. B. Ustinov, A. V. Drozdovskii, A. A. Nikitin, and B. A. Kalinikos, "Spinwave band-pass filters based on yttrium iron garnet films for tunable microwave photonic oscillators," Journal of Physics: Conference Series 661 , 012058 (2015).
${ }^{26}$ Y. Tabuchi, S. Ishino, T. Ishikawa, R. Yamazaki, K. Usami, and Y. Nakamura, "Hybridizing Ferromagnetic Magnons and Microwave Photons in the Quantum Limit," Physical Review Letters 113, 083603 (2014).

${ }^{27}$ S. Kosen, A. F. van Loo, D. A. Bozhko, L. Mihalceanu, and A. D. Karenowska, "Microwave magnon damping in YIG films at millikelvin temperatures," APL Materials 7, 101120 (2019).

${ }^{28} \mathrm{M}$. Pfirrmann, I. Boventer, A. Schneider, T. Wolz, M. Kläui, A. V. Ustinov, and M. Weides, "Magnons at low excitations: Observation of incoherent coupling to a bath of two-level systems," Physical Review Research 1, 032023 (2019).

${ }^{29}$ S. Probst, F. B. Song, P. A. Bushev, A. V. Ustinov, and M. Weides, "Efficient and robust analysis of complex scattering data under noise in microwave resonators," Review of Scientific Instruments 86, 024706 (2015).

${ }^{30}$ qkitgroup, "Qkit - a quantum measurement suite in python,"

${ }^{31}$ C. Müller, J. H. Cole, and J. Lisenfeld, "Towards understanding two-levelsystems in amorphous solids: Insights from quantum circuits," Reports on Progress in Physics 82, 124501 (2019).

${ }^{32} \mathrm{~N}$. Wiener, "Generalized harmonic analysis," Acta Mathematica 55, 117258 (1930).

${ }^{33} \mathrm{P}$. Welch, "The use of fast Fourier transform for the estimation of power spectra: A method based on time averaging over short, modified periodograms," IEEE Transactions on Audio and Electroacoustics 15, 70-73 (1967)

${ }^{34}$ J. Gao, J. Zmuidzinas, B. A. Mazin, H. G. LeDuc, and P. K. Day, "Noise properties of superconducting coplanar waveguide microwave resonators," Applied Physics Letters 90, 102507 (2007).

${ }^{35}$ S. Kumar, J. Gao, J. Zmuidzinas, B. A. Mazin, H. G. LeDuc, and P. K. Day, "Temperature dependence of the frequency and noise of superconducting coplanar waveguide resonators," Applied Physics Letters 92, 123503 (2008)

${ }^{36}$ C. Neill, A. Megrant, R. Barends, Y. Chen, B. Chiaro, J. Kelly, J. Y. Mutus, P. J. J. O'Malley, D. Sank, J. Wenner, T. C. White, Y. Yin, A. N. Cleland, and J. M. Martinis, "Fluctuations from edge defects in superconducting resonators," Applied Physics Letters 103, 072601 (2013).

${ }^{37}$ J. Burnett, L. Faoro, I. Wisby, V. L. Gurtovoi, A. V. Chernykh, G. M. Mikhailov, V. A. Tulin, R. Shaikhaidarov, V. Antonov, P. J. Meeson, A. Y. Tzalenchuk, and T. Lindström, "Evidence for interacting two-level systems from the $1 / f$ noise of a superconducting resonator," Nature Communications 5, ncomms5119 (2014)

${ }^{38}$ J. D. Brehm, A. Bilmes, G. Weiss, A. V. Ustinov, and J. Lisenfeld, "Transmission-line resonators for the study of individual two-level tunneling systems," Applied Physics Letters 111, 112601 (2017).

${ }^{39}$ P. Dutta and P. M. Horn, "Low-frequency fluctuations in solids: $1 / f$ noise," Reviews of Modern Physics 53, 497-516 (1981).

${ }^{40} \mathrm{~L}$. Faoro and L. B. Ioffe, "Interacting tunneling model for two-level systems in amorphous materials and its predictions for their dephasing and noise in superconducting microresonators," Physical Review B 91, 014201 (2015).

${ }^{41}$ R. Bos, S. de Waele, and P. M. T. Broersen, "Autoregressive spectral estimation by application of the Burg algorithm to irregularly sampled data," IEEE Transactions on Instrumentation and Measurement, 51 (6) (2002).

${ }^{42}$ G. E. P. Box, G. M. Jenkins, G. C. Reinsel, and G. M. Ljung, Time Series Analysis: Forecasting and Control (John Wiley \& Sons, 2015).

${ }^{43} \mathrm{G}$. U. Yule, "On a method of investigating periodicities disturbed series, with special reference to Wolfer's sunspot numbers," Philosophical Transactions of the Royal Society of London. Series A, Containing Papers of a Mathematical or Physical Character 226, 267-298 (1927).

${ }^{44}$ G. T. Walker, "On periodicity in series of related terms," Proceedings of the Royal Society of London. Series A, Containing Papers of a Mathematical and Physical Character 131, 518-532 (1931). 
(a)

(b) Frequency sweep
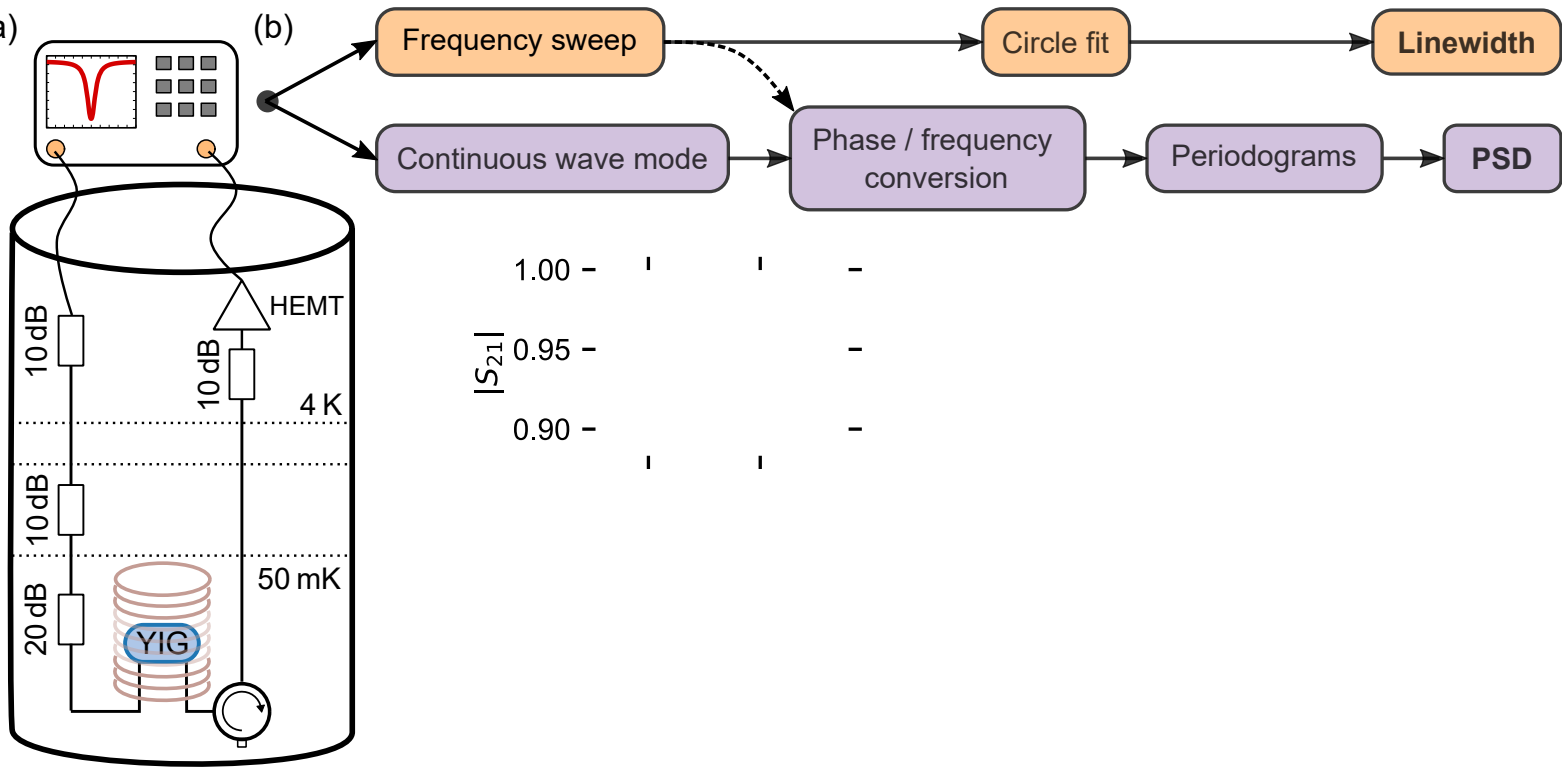

ज̃ $0.95-$ conversion

$0.90-$

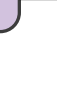



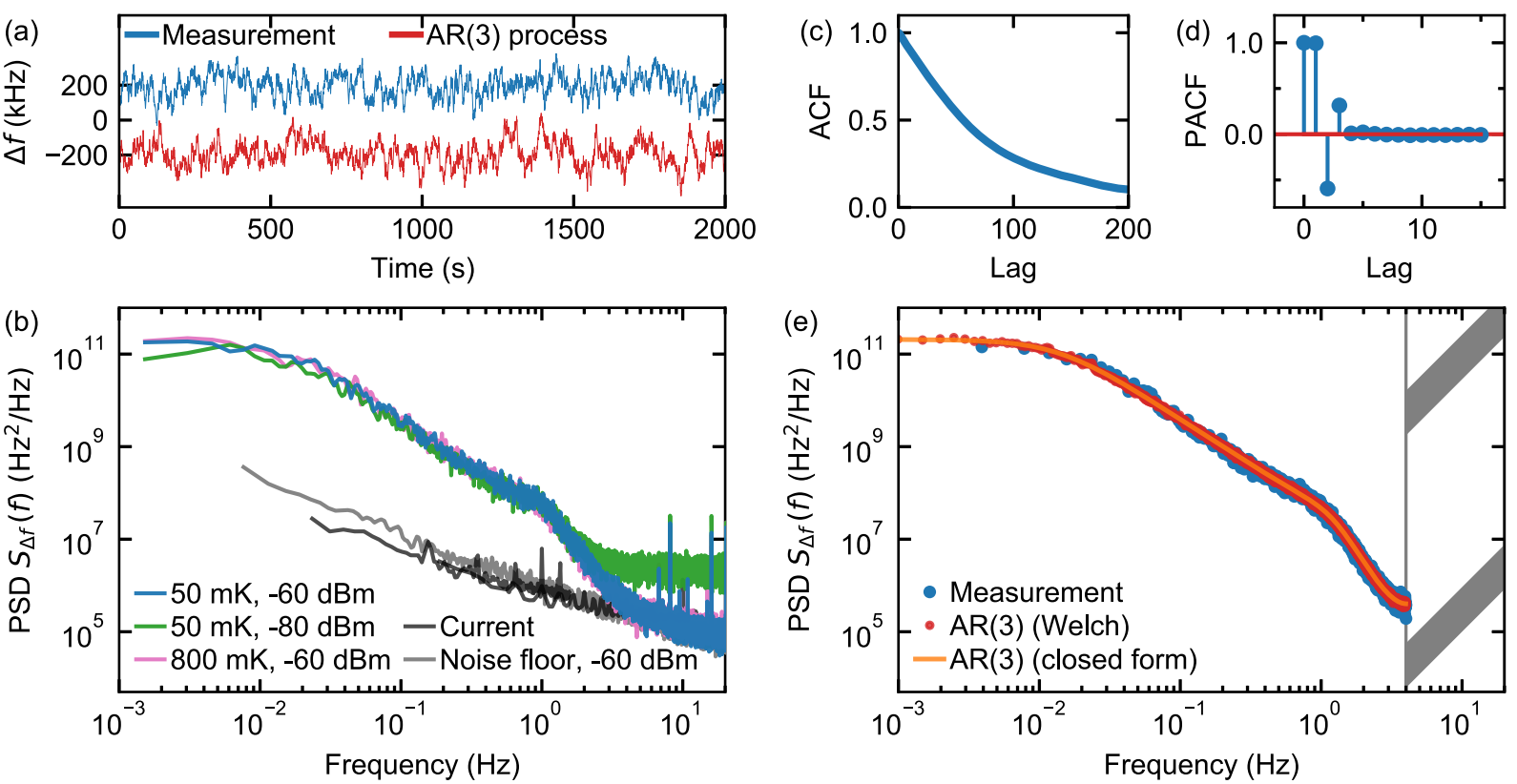